\title{
Hallazgos de ultrasonido transfontanelar en neonatos y lactantes menores con alto riesgo biológico.
}

Transfontanellar ultrasound findings in neonates and young infants with high biological risk.

https://doi.org/10.54139/salus.v25i2.57

María José Benavides Colina ${ }^{1}$ (D) Germán Perdomo Oramas ${ }^{1}$ (D) Harold Guevara Rivas ${ }^{2}$ (D) María Elizabeth García Torres ${ }^{1}$ (D) Ángel Riera García1 1 D

\section{RESUMEN}

Introducción: Los neonatos y lactantes menores de alto riesgo están expuestos a un amplio espectro de lesiones cerebrales que pueden ser detectadas por ultrasonido transfontanelar. Métodos: Se analizaron los hallazgos del ultrasonido transfontanelar y se correlacionaron con las complicaciones perinatales, la edad gestacional y el diagnóstico clínico del paciente. Se realizó un estudio no experimental, prospectivo, transversal, descriptivo y comparativo. La muestra estuvo comprendida por 90 neonatos y lactantes menores con criterio de alto riesgo, que cumplieron con los criterios de inclusión. Resultados: Los hallazgos evidenciados en el estudio de ultrasonido fueron $72,1 \%$ resultaron normales, $27,7 \%$ de los pacientes tuvo algún hallazgo patológico, encontrando hemorragia de la matriz germinal grado I $(6,6 \%)$, quistes subependimarios unilaterales $(6,6 \%)$ y bilateral $(1,1 \%)$, hidrocefalia $(4,4 \%)$, agenesia del cuerpo calloso con colpocefalia $(2,2 \%)$, hemorragia intraparenquimatosa, hidranencefalia, quiste aracnoideo, calcificaciones periventriculares y talámicas, así como vasculopatía lenticuloestriada y ventriculitis $(1,1 \%$ cada una). Discusión: El ultrasonido transfontanelar es el método de neuroimagen de elección inicial para los recién nacidos y lactantes menores de alto riesgo, ya que es libre de radiación ionizante, permitiendo diagnosticar y realizar seguimiento de patologías cerebrales en esta población de riesgo.

Palabras clave: Alto riesgo, neonatos, lactantes menores, ultrasonido transfontanelar.

1 Servicio de Radiología e Imagenología, Ciudad Hospitalaria Dr. Enrique Tejera. Valencia, Estado Carabobo. Venezuela.

2 Departamento de Salud Pública de la Facultad de Ciencias de la Salud de la Universidad de Carabobo. Valencia, Venezuela

Autor de correspondencia: María José Benavides (DD

E-mail: mariajbenavides90@gmail.com

Recibido: 03-02-2021

Aprobado:18-07-2021
Introduction: High risk neonates and young infants are exposed to a wide spectrum of brain lesions that can be detected by transfontanellar ultrasound. Transfontanellar ultrasound findings were analyzed and correlated with perinatal complications, gestational age, and clinical diagnosis of the patient. Methods: A non-experimental, prospective, cross-sectional, descriptive, and comparative study was carried out. The sample consisted of 90 neonates and young infants with high-risk criteria, who met the inclusion criteria. Results: Findings from the ultrasound study of the patients indicated that $72.1 \%$ were normal, $27.7 \%$ had some kind of pathology which included grade I germinal matrix hemorrhage $(6.6 \%)$, unilateral subependymal cysts $(6.6 \%)$ and bilateral $(1.1 \%)$, hydrocephalus $(4.4 \%)$, agenesis of the corpus callosum with colpocephaly $(2.2 \%)$, intraparenchymal hemorrhage, hydranencephaly, arachnoid cyst, periventricular and thalamic calcifications, as well as lenticulostriate vasculopathy and ventriculitis (1.1\% each). Discussion: Transfontanellar ultrasound is the first-choice neuroimaging method for high-risk newborns and young infants; it is free of ionizing radiation, allowing the diagnosis and monitoring of brain pathologies in this vulnerable population.

Key words: high risk, neonates, young infants, transfontanellar ultrasound.

\section{INTRODUCCIÓN}

El desarrollo de la Neonatología ha conseguido aumentar la supervivencia de pacientes que presentan una gran morbilidad, al punto de ser considerados "recién nacidos de alto riesgo", debido a que sus antecedentes prenatales, perinatales o postnatales. Desde la concepción hasta los primeros años de vida ocasionan anomalías, transitorias o definitivas, que pueden alterar la adecuada maduración del sistema nervioso central, repercutiendo en su desarrollo motor, sensorial, cognitivo o conductual, pudiendo conllevar a retraso y/o afección de su crecimiento y desarrollo norma Estos factores son también denominados alto riesgo biológico por algunos autores (1).

Dentro de los factores de riesgo hay un primer grupo en el que se encuentran los neonatos con riesgo orgánico, aquellos con: trastornos cromosómicos, malformaciones congénitas mayores que precisaron cirugía, prematuridad (edad gestacional menor de 32 semanas), bajo peso al nacer (menor a 1500 gramos), peso inferior a 2 DS para su edad gestacional, asfixia severa, Apgar a los 5 minutos $\leq 3$ puntos, ventilación mecánica prolongada, retraso de crecimiento intrauterino, infecciones congénitas y del sistema nervioso 
central, encefalopatía hipóxico isquémica, convulsiones neonatales, enfermedad congénita del metabolismo susceptible de ocasionar déficit neurológico, hiperbilirrubinemia que precisan exanguinotransfusión, policitemia, cardiopatías congénitas, displasia broncopulmonar, entre otros (2).

El segundo grupo pertenece a los neonatos con riesgo psicosocial: hijos de padres con bajo nivel socioeconómico, retraso mental o enfermedad psiquiátrica, abuso de sustancias tóxicas, antecedente de maltrato o abandono de otros hijos, madre menor de 20 años o que no realizó seguimiento prenatal y familias monoparentales sin apoyo. La detección precoz de cualquiera de estas anomalías del desarrollo tanto orgánicas como psicosociales permitirá una intervención temprana que pueda modificar favorablemente el futuro del niño que las padece y de esta forma mejorar su adaptación social y familiar (2).

Las consecuencias más comunes y graves que pueden presentar los pacientes con alto riesgo son los trastornos del sistema nervioso central, como la hemorragia cerebral, hidrocefalia posthemorrágica, leucomalacia periventricular e infarto cerebral. Estos trastornos presuponen un alto riesgo de deficientes resultados neurológicos a largo plazo.

Los prematuros con hemorragia intraventricular subependimaria grado I o II pueden tener alteraciones en el neurodesarrollo. La hemorragia intraventricular de grado III o grado IV se asocia con los resultados del desarrollo neurológico más desfavorables, pero el grado de prematuridad y la presencia de otras comorbilidades, pueden ser también, los principales contribuyentes a discapacidades graves a largo plazo. Los bebés que han tenido hemorragia intracraneal de cualquier grado siempre deben tener un seguimiento del neurodesarrollo.

Durante el ingreso en las Unidades de Cuidados Intensivos Neonatales (UCIN) los niños reciben cuidados especializados, permitiendo que muchos recién nacidos prematuros o con malformaciones mayores sobrevivan, La reducción de la mortalidad no es necesariamente igual a la reducción en las tasas de discapacidad, pues se genera un aumento en la población de niños con necesidades complejas. Los déficits cognitivos, sin grandes déficits motores, son ahora las secuelas del neurodesarrollo más frecuentes. A medida que crece el número total de sobrevivientes, aumenta el riesgo potencial de morbilidad en el desarrollo neurológico por déficits neurocognitivos, que pueden no ser evidenciables en etapas tempranas, identificarse sólo con estudios de seguimiento a largo plazo.

Es fundamental, entonces, continuar asistiendo a estos pacientes para conocer su evolución a largo plazo, surgiendo así los programas de consultas de seguimiento, durante los cuales se han revelado problemas de desarrollo neurológico sutiles, que requieren evaluaciones e intervenciones complejas.
La atención al neonato críticamente enfermo en el marco de las Unidades de Cuidados Intensivos Neonatales (UCIN), así como durante el seguimiento de los pacientes detectados como alto riesgo, debe incluir el uso de técnicas de imagen que permitan la más adecuada aproximación diagnóstica al trastorno del paciente, siendo el ultrasonido transfontanelar (UT) una herramienta excelente para detectar las anomalías cerebrales más frecuentes en neonatos prematuros y a término, para estudiar la evolución de las lesiones y seguir la maduración cerebral en los lactantes menores.

Desde su introducción clínica a finales de la década de 1970, el UT se ha utilizado ampliamente para detectar anomalías congénitas y lesiones cerebrales adquiridas durante el período neonatal, convirtiéndose en un método indispensable en el estudio de estas patologías.

El ultrasonido ofrece ventajas sobre otras modalidades de imagen, como es la tomografía computarizada. Es menos costoso, la visualización de las imágenes es en tiempo real, se libera al paciente de ser expuesto a radiación ionizante, lo que permite obtener imágenes en serie, no requiere sedación, es fácil de usar y su portabilidad permite la evaluación junto a la cama en pacientes gravemente enfermos que no pueden ser trasladados a las salas de radiología para obtener las imágenes.

Con el tiempo, la calidad del ultrasonido transfontanelar ha mejorado gracias a técnicas avanzadas con una resolución más alta, un procesamiento de imágenes más rápido y una pantalla y copia de seguridad digital. Las estructuras cerebrales importantes se pueden visualizar adecuadamente usando configuraciones óptimas.

Tradicionalmente las imágenes se obtienen a través de la fontanela anterior. Este enfoque es menos adecuado para la evaluación de estructuras infratentoriales porque están ubicadas lejos del transductor y el tentorio muy ecogénico impide su evaluación. El uso de transductores lineales de alta frecuencia a través de ventanas acústicas alternativas y configuraciones adaptadas, también proporciona acceso a estas regiones del cerebro. Ejemplos de estas ventanas acústicas suplementarias son las fontanelas lambdoideas (posterior), mastoidea y lateral (temporal). Hasta el momento, sin embargo, solo unas pocas UCIN y hospitales pediátricos en general, utilizan estas ventanas acústicas adicionales de forma rutinaria.

A estas mejoras se le añade la técnica Doppler que resulta un valioso instrumento para detectar la permeabilidad de los vasos intracraneales, así como para obtener velocidades de flujo e índices de resistencias en las arterias cerebrales (3).

Otra técnica utilizada para obtener a la neuroimagen es la resonancia magnética (RM) que proporciona una excelente calidad de imagen, pero su uso clínico en las UCIN e incluso para lactantes menores, está limitado debido a problemas de logística y seguridad y a que en algunos casos amerita 
el uso de sedación. Cuando el equipo moderno se combina con una técnica de imagen completa, el ultrasonido ofrece resultados similares a los de la RM en términos de sensibilidad y capacidad para dirigir el manejo inicial.

En el 2014, en Austria, Riccabona et al. (4) llevaron a cabo un estudio en el que resaltan la importancia del UT para el diagnóstico de afecciones neurológicas que influyen significativamente en la morbilidad y la mortalidad de neonatos y lactantes. A pesar de que con la introducción de la Tomografía Computada (TC) y la RM, el ultrasonido transfontanelar neonatal se ha considerado cada vez más solo como una técnica que ofrece información de orientación y no entrega mucha información relevante. Esto se debe en parte a un bajo rendimiento del uso del ultrasonido, ya sea por la disponibilidad limitada de equipos modernos o por la falta de experiencia especializada en la realización y lectura de exploraciones neurosonográficas.

Con su revisión Riccabona intenta resaltar el valor y el potencial del ultrasonido en la exploración del cerebro neonatal y por otro lado, en la evaluación de las imágenes de la médula espinal. Su objetivo es alentar a los radiólogos pediátricos a reorientar los algoritmos y las habilidades de imagen hacia el potencial de la neurosonografía moderna, particularmente en vista de la eficacia, considerando la creciente presión económica, así como la buena disponibilidad del ultrasonido en cualquier momento.

La principal indicación para el UT es la demostración o exclusión de hemorragia intracraneal en un recién nacido prematuro. La técnica se utiliza adicionalmente para el seguimiento de las complicaciones relacionadas con la hemorragia intraventricular y para buscar hallazgos evolutivos relacionados con la isquemia. Otras indicaciones más amplias incluyen la demostración de anomalías estructurales congénitas, lesiones vasculares intracraneales y también su uso como una herramienta de detección simple en la exclusión de patología intracraneal gruesa (5).

En el 2013 en Nigeria, Marchie et al. (6), realizaron una investigación del cerebro infantil con o sin hidrocefalia mediante ecografía transfontanelar anterior, con el objetivo de sugerir un sitio ventricular fácil y simple, determinado según el plano de medición básico y la dimensión normal, correlacionándolo con el tamaño de los lactantes para la evaluación comparativa de lactantes hidrocéfalos que sea reproducible en el seguimiento. Se encontró que el UT fue muy útil en el estudio de hidrocefalia en lactantes.

En Corea en el 2018, Choi et al. (7), llevaron a cabo un estudio para establecer los patrones de lesión isquémica en imágenes cerebrales en neonatos con meningitis estreptocócica del grupo B. Se encontró que ciertos patrones de lesión isquémica son comúnmente reconocidos en imágenes cerebrales de pacientes con meningitis neonatal y esta complicación isquémica puede modificar los procesos de la enfermedad y contribuir a resultados neurológicos deficientes. En 6 lactantes (75\%) la ecografía craneal mostró ecogenicidad focal o difusa, lo que sugiere una lesión hipóxico-isquémica en los ganglios basales compatibles con meningitis, demostrando la aplicabilidad del ultrasonido en la detección de los procesos isquémicos, incluso los producidos por agentes infecciosos.

En España, en el 2016, Llorens y Moreno (8) refieren que se han encontrado limitaciones del ultrasonido en el estudio de la patología traumática obstétrica, la valoración de la patología malformativa compleja y el daño de la sustancia blanca. No obstante, señalan que, con los conocimientos básicos de neurología neonatal, el equipamiento apropiado y una técnica cuidadosa que incluya el uso de distintas fontanelas, es un método fiable que permite el diagnóstico y seguimiento de patologías tanto congénitas como adquiridas en el neonato.

En el año 2013, Ciambra et al. (9), analizando las limitaciones sobre la exactitud del UT en la detección de lesiones leves de sustancia blanca en recién nacidos, encontraron que la RM parece ser más confiable para detectar anomalías leves o moderadas de la sustancia blanca. Así, el ultrasonido en serie puede proporcionar información valiosa sobre los cambios tempranos de la sustancia blanca, como las ecogenicidades aumentadas que podrían conducir a una lesión difusa. Consideran que el UT en serie en los recién nacidos prematuros es esencial y no puede reemplazarse con RM.

En vista de la considerable cantidad de pacientes que cursan con patologías o condiciones contempladas en los denominados factores de alto riesgo, se realizó un estudio que permita evaluar esta población mediante el ultrasonido transfontanelar, tanto para el diagnóstico inicial, como para el seguimiento de la patología ya conocida.

\section{MATERIALES Y MÉTODOS}

Se analizaron los hallazgos de UT en neonatos y lactantes menores con alto riesgo atendidos en el Hospital de Niños "Dr. Jorge Lizárraga", en el periodo Agosto 2019 Mayo 2020; se distribuyó la muestra según sexo, edad, antecedentes perinatales y edad gestacional; se clasificaron los pacientes según el diagnóstico clínico; se describieron los hallazgos ultrasonográficos y estos se asociaron con los antecedentes perinatales y el diagnóstico clínico; se relacionó la edad gestacional con las alteraciones del ultrasonido y con la existencia de variante anatómica; se midió el índice de resistencia y se evaluó su relación con el diagnóstico clínico, la edad gestacional y la edad de vida. Se trató de un estudio de campo no experimental, prospectivo, con un diseño de investigación transversal, descriptivo, comparativo. La población fue constituida por neonatos y lactantes menores de alto riesgo que fueron atendidos en el Hospital de Niños "Dr. Jorge Lizarraga”, en el periodo entre agosto 2019 y mayo 2020. 
La muestra estudiada fue no probabilística, intencional, constituida por 90 recién nacidos y lactantes menores que cumplían con los criterios de inclusión de contar con fontanelas permeables, con diagnóstico que los designara con alto riesgo, que estaban ingresados en el retén del Servicio de Neonatología, atendidos en la emergencia pediátrica, o en consulta externa de Neurología Pediátrica del mismo hospital de niños mencionado, y que ameritaron la realización del UT, como procedimiento diagnóstico o de control; sin distinción de sexo. Los criterios de exclusión fueron inestabilidad clínica que impidiera el traslado del paciente.

Se solicitó el consentimiento informado a los padres o representantes de los pacientes, con un documento escrito, informándose de las características del estudio, el procedimiento a realizar y los beneficios del mismo. La recolección de datos se realizó mediante la anamnesis, donde se indagaron las variables a estudiar, como los datos del paciente, sexo, edad, edad gestacional, antecedentes perinatales y familiares y diagnóstico clínico.

El ultrasonido transfontanelar en compañía de padres o representantes, colocando al neonato o lactante sobre sus piernas. La técnica de estudio se realizó usando un equipo de ultrasonido marca Sonoscape $\AA$, modelo $\mathrm{S} 12$, con transductores convex de 3 a $9 \mathrm{MHz}$ y lineal de 5 a 14 $\mathrm{MHz}$, mediante los cuales se realizó contacto directo sobre la fontanela anterior permeable como ventana acústica, o en caso de ser necesario a través de la fontanela posterior y mastoidea, con aplicación de gel especial para ultrasonido. Se hizo uso del modo B y del preset establecido para el estudio del cerebro pediátrico, para obtener las imágenes bajo la configuración indicada. La evaluación fue llevada a cabo por un Médico Residente del Postgrado de Radiología bajo la supervisión de un Médico Especialista en Radiología.

Durante su realización se analizaron las imágenes secuenciales obtenidas en cada corte, se visualizó detalladamente la anatomía cerebral, en búsqueda de patrones de alteraciones de ecogenicidad, estructurales o morfológicas que pudieran indicar algún proceso patológico para describir y localizar los hallazgos, los cuales fueron almacenados para disponer de un archivo digital. (10)

En caso de evidenciar casos de hemorragia de la matriz germinal, se subdividieron en grados, correspondiendo el grado I a la hemorragia subependimaria, el grado II a la extensión intraventricular sin hidrocefalia, el grado III a la hemorragia intraventricular con hidrocefalia y el grado IV a hemorragia intraparenquimatosa con o sin hidrocefalia (11-14).

La mayoría de los pacientes ameritaban el estudio por presentar múltiples diagnósticos clínicos, siendo seleccionada la patología de mayor severidad y de afectación conocida en el sistema nervioso central. Tomando en cuenta que, de las patologías seleccionadas, los pacientes presentaban sintomatologías de alteración del sistema nervioso central, desde convulsiones, hasta diversos grados de déficit neurológico.

Se evaluaron los datos recolectados y, posteriormente se ordenaron, tabularon y analizaron con el paquete estadístico PAST versión 3.17, mediante estadística descriptiva e inferencial. Los resultados se presentan en cuadros de distribución de frecuencias absolutas y relativas, así como de asociación. Las variables cuantitativas se describen con la mediana, el rango intercuartil y los percentiles 25 y 75 , en vista de no adaptarse a la distribución normal, según la prueba de Kolmogorov-Smirnov.

Para el análisis de las variables cualitativas se aplicó la prueba estadística de Chi-cuadrado $(X 2)$ con corrección de Yates en tablas tetracóricas y el $x 2$ de Pearson en tablas $3 x$ 2. Para la comparación de las variables numéricas entre los pacientes con ecosonograma patológico o no se realizó la prueba no paramétrica de Mann-Whitney (MW). Se aplicó el coeficiente de correlación por rangos de Spearman $(\rho)$ para relacionar la edad gestacional, la edad del niño y el índice de resistencia de la arteria pericallosa, asumiendo para todas las pruebas un nivel de significancia estadística de $\mathrm{P}<0,05$.

\section{RESULTADOS}

Tabla 1. Distribución de los pacientes pediátricos estudiados según sexo, edad, antecedentes perinatales y edad gestacional

\begin{tabular}{|l|c|c|}
\hline Sexo & Frecuencia & Porcentaje \\
\hline Masculino & 53 & $58,9^{*}$ \\
\hline Femenino & 37 & 41,1 \\
\hline Edad del recién nacido o lactante menor (días) \\
\hline 3 a 57 & 76 & $84,4^{*}$ \\
\hline 58 a 102 & 5 & 5,6 \\
\hline 113 a 157 & 2 & 2,2 \\
\hline 168 a 222 & 2 & 2,2 \\
\hline 223 a 274 & 4 & 4,4 \\
\hline 275 a 339 & 1 & 1,1 \\
\hline Edad gestacional (semanas) & & \\
\hline 32 a 36 & 21 & 23,3 \\
\hline 37 a 40 & 66 & $73,3^{*}$ \\
\hline 41 & 3 & 3,3 \\
\hline Madurez del recién nacido & & $76,7^{*}$ \\
\hline Pretérmino tardío & 14 & 15,6 \\
\hline Pretérmino moderado & 7 & \\
\hline A término & 69 & \\
\hline Estadicame & & \\
\hline
\end{tabular}

*Estadísticamente significativo 
De la muestra estudiada $58,9 \%$ (53) fueron de sexo masculino, con diferencias estadísticamente significativas $(Z=2,24 ; P=0,01) ; 84,4 \%$ (76) tenían entre 3 y 57 días de vida, con predominio estadísticamente significativo $(Z=9,09$; $\mathrm{P}=0,00)$. La edad del recién nacido o lactante menor tuvo valor mínimo 3 días, máximo 330 días, mediana 25 días, el percentil 25 se ubicó en 15 días y el percentil 75 en 44,25 días; $73,3 \%$ (66) tuvieron edad gestacional entre 37 y 40 semanas, con relevancia significativa $(Z=6,11 ; P=0,00)$. La edad gestacional tuvo valor mínimo 32 semanas, máximo 41 semanas +4 días, la mediana 38 semanas, el percentil 25 se ubicó en 37 semanas y el percentil 75 en 39 semanas. El 76,7\% (79 productos de la concepción) se encontraban a término, con relevancia estadísticamente significativa $(Z=9,99 ; P=0,00)$ (Tabla1).

Tabla 2. Distribución de los pacientes pediátricos estudiados según las complicaciones gestacionales

\begin{tabular}{|l|c|c|}
\hline Complicación gestacional & Frecuencia & Porcentaje \\
\hline Infección genitourinaria & 13 & 14,4 \\
\hline Preeclampsia & 7 & 7,7 \\
\hline $\begin{array}{l}\text { Infección de transmisión sexual } \\
\text { VDRL reactivo }\end{array}$ & 4 & 4,4 \\
\hline Embarazo gemelar & 3 & 3,3 \\
\hline Oligohidramnios & 2 & 2,2 \\
\hline Paludismo & 1 & 1,1 \\
\hline Epilepsia & 1 & 1,1 \\
\hline Síndrome convulsivo & 1 & 1,1 \\
\hline Trauma obstétrico & 1 & 1,1 \\
\hline Doble circular de cordón. Edad & 1 & 1,1 \\
\hline materna avanzada & 1 & 1,1 \\
\hline Cardiopatía & 1 & 1,1 \\
\hline Hipertensión arterial & 52 & 1,1 \\
\hline Eclampsia & & 57,7 \\
\hline Edad materna avanzada (mayor & & 1,1 \\
\hline Sin complicaciones conocidas & & 1 \\
\hline
\end{tabular}

El 57,7\% (52) pacientes no presentaron complicaciones gestacionales, con predominio estadísticamente significativo $(Z=1,94 ; P=0,03) ; 14,4 \%$ (13 casos) presentaron infecciones genitourinarias en distintos trimestres del embarazo. $7,7 \%$ (7 casos), pre-eclampsia. 4,4\% (4 casos) infección de transmisión sexual con VDRL reactivo, 3,3\% (3 mujeres) correspondían a embarazo gemelar, 2,2\% (2 casos) oligrohidramnios (Tabla 2).
En la Tabla 3 se puede observar que los diagnósticos clínicos más frecuentes fueron sepsis punto de partida a precisar $23,3 \%$ (21 pacientes) y sepsis punto de partida sistema nervioso central (SNC)15,5\% (14 pacientes).

Tabla 3. Distribución de los pacientes pediátricos estudiados según diagnóstico clínico.

\begin{tabular}{|c|c|c|}
\hline Diagnóstico clínico & Frecuencia & Porcentaje \\
\hline Sepsis punto de partida a precisar & 21 & 23,3 \\
\hline Sepsis punto de partida SNC & 14 & 15,5 \\
\hline Asfixia perinatal & 8 & 8,8 \\
\hline Infección SNC & 8 & 8,8 \\
\hline Síndrome convulsivo no febril & 5 & 5,5 \\
\hline Desnutrición & 4 & 4,4 \\
\hline LUES congénito & 4 & 4,4 \\
\hline Síndrome convulsivo febril & 4 & 4,4 \\
\hline Sepsis punto de partida enteral & 3 & 3,3 \\
\hline Ciemopatía del SNC (perinatal) & 3 & 3,3 \\
\hline Traumatismo por caída de altura & 2 & 2,2 \\
\hline $\begin{array}{l}\text { Infección asociada a cuidados de la } \\
\text { salud }\end{array}$ & 2 & 2,2 \\
\hline Ultrasonido perinatal patológico & 1 & 1,1 \\
\hline Error innato del metabolismo & 1 & 1,1 \\
\hline $\begin{array}{l}\text { Fenotipo Down. Sepsis punto } \\
\text { partida SNC }\end{array}$ & 1 & 1,1 \\
\hline Infección respiratoria baja & 1 & 1,1 \\
\hline Otitis media aguda supurativa & 1 & 1,1 \\
\hline Cierre precoz de fontanela & 1 & 1,1 \\
\hline Sepsis punto de partida respiratorio & 1 & 1,1 \\
\hline Shock séptico & 1 & 1,1 \\
\hline Síndrome dismórfico & 1 & 1,1 \\
\hline $\begin{array}{l}\text { Síndrome dismórfico. Cardiopatía } \\
\text { congénita acianógena }\end{array}$ & 1 & 1,1 \\
\hline $\begin{array}{l}\text { Síndrome dismórfico. } \\
\text { Craneosinostosis }\end{array}$ & 1 & 1,1 \\
\hline $\begin{array}{l}\text { Síndrome dismórfico. Sepsis punto } \\
\text { de partida a precisar }\end{array}$ & 1 & 1,1 \\
\hline
\end{tabular}


Tabla 4. Distribución de los pacientes pediátricos estudiados según los hallazgos del ultrasonido y variantes anatómicas

\begin{tabular}{|c|c|c|}
\hline Hallazgos en el ultrasonido & Frecuencia & Porcentaje \\
\hline $\begin{array}{l}\text { Hemorragia de la matriz germinal } \\
\text { (grado I) }\end{array}$ & 5 & 5,5 \\
\hline Quiste subependimario unilateral & 6 & 6,6 \\
\hline Hidrocefalia & 3 & 3,3 \\
\hline $\begin{array}{l}\text { Agenesia del cuerpo calloso. } \\
\text { Colpocefalia }\end{array}$ & 2 & 2,2 \\
\hline $\begin{array}{l}\text { Quistes subependimarios } \\
\text { bilaterales }\end{array}$ & 1 & 1,1 \\
\hline $\begin{array}{l}\text { Hemorragia de la matriz germinal } \\
\text { (grado I) con degeneración quística } \\
\text { subependimaria }\end{array}$ & 1 & 1,1 \\
\hline $\begin{array}{l}\text { Hemorragia intraparenquimatosa } \\
\text { talámica }\end{array}$ & 1 & 1,1 \\
\hline $\begin{array}{l}\text { Hidrocefalia con asimetría de } \\
\text { ventrículos laterales }\end{array}$ & 1 & 1,1 \\
\hline $\begin{array}{l}\text { Hidranencefalia. Vasculopatía } \\
\text { lenticuloestriada }\end{array}$ & 1 & 1,1 \\
\hline Quiste aracnoideo & 1 & 1,1 \\
\hline Calcificaciones periventriculares & 1 & 1,1 \\
\hline Calcificaciones en tálamo derecho & 1 & 1,1 \\
\hline $\begin{array}{l}\text { Vasculopatía lentículoestriada } \\
\text { aislada }\end{array}$ & 1 & 1,1 \\
\hline Ventriculitis & 1 & 1,1 \\
\hline Normal & 65 & 72,2 \\
\hline \multicolumn{3}{|l|}{ Variante anatómica } \\
\hline No & 61 & 67,8 \\
\hline Sí & 29 & 32,2 \\
\hline
\end{tabular}

La tabla 4 muestra que los hallazgos más frecuentes en el ultrasonido fueron la hemorragia de la matriz germinal grado I, $5,5 \%$ (5 pacientes) y degeneración quística subependimaria $1,1 \%$ (1 paciente) [Fig. 1], quiste subependimario unilateral $6,6 \%$ (6 pacientes), quiste subependimario bilateral $1.1 \%$ (1 paciente). Hidrocefalia en total 4,4\% (4 pacientes) [Fig. 2], tomando en cuenta 1 caso de hidrocefalia con asimetría de los ventrículos laterales. El 72,2\% (65 niños) presentaron un ultrasonido normal. La variante anatómica encontrada fue el cavum del septum pellucidum con $32,2 \%$ (29 pacientes). También se evidenciaron alteraciones como agenesia del cuerpo calloso y colpocefalia $2,2 \%$ (2 pacientes), hidranencefalia asociada a vasculopatía lentculoestriada 1,1\% (1 paciente) [Fig. 3], así como quiste aracnoideo en región prepontina y supraselar 1,1\% (1 paciente) [Fig. 4].

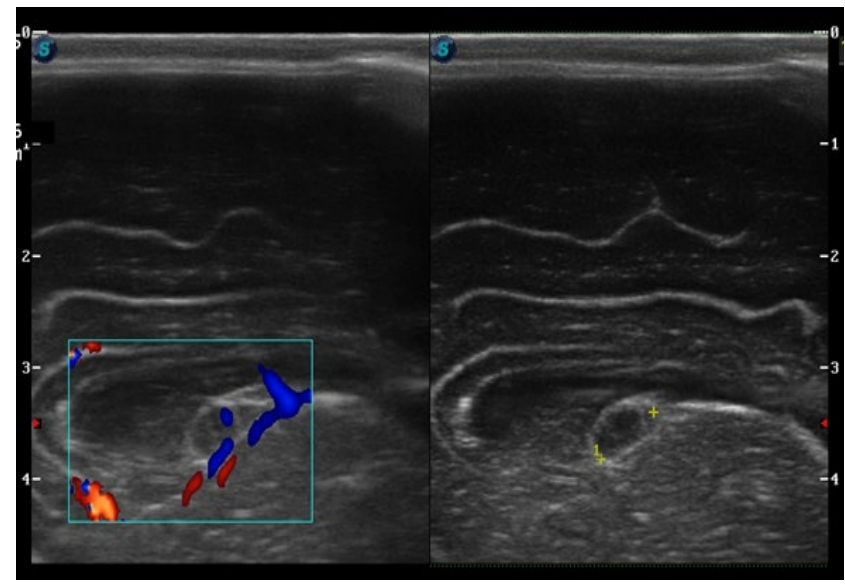

Fig. 1. Imágenes sagitales que muestran lesión hipoecoica, ovalada, de pared ecogénica bien definida, con señal Doppler periférica, localizada en surco caudotalámico, correspondiente a resolución de hemorragia subependimaria con transformación quística. Benavides, María 2020.

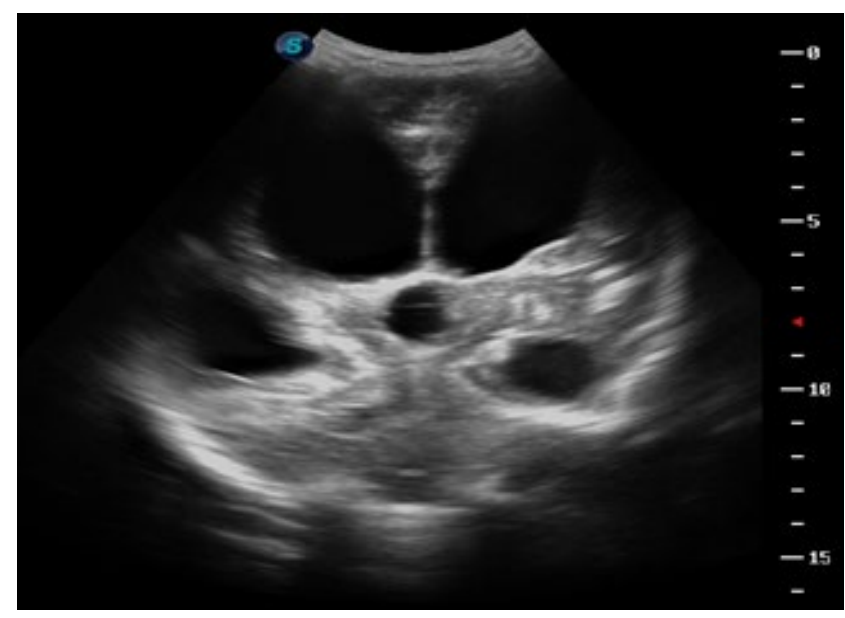

Fig. 2. Imagen coronal en la que se evidencia dilatación de ventrículos laterales y tercer ventrículo. Benavides, María 2019.

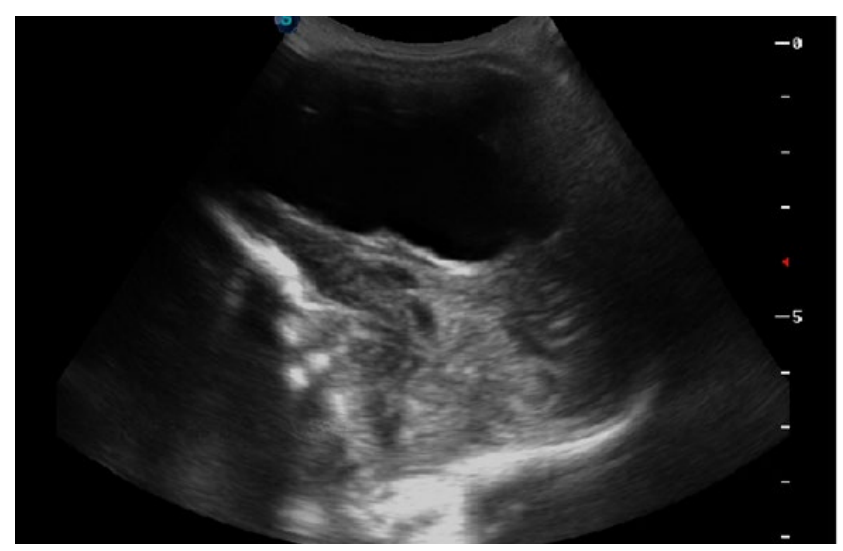

Fig. 3. Se evidencia ausencia de lóbulos parietales y parcial de frontales, cuyos espacios son ocupados por líquido cefalorraquídeo. Lóbulos occipitales, tallo cerebral y cerebelo con morfología adecuada. Hallazgos de hidranencefalia. Benavides M, Garcia M. 2019. 


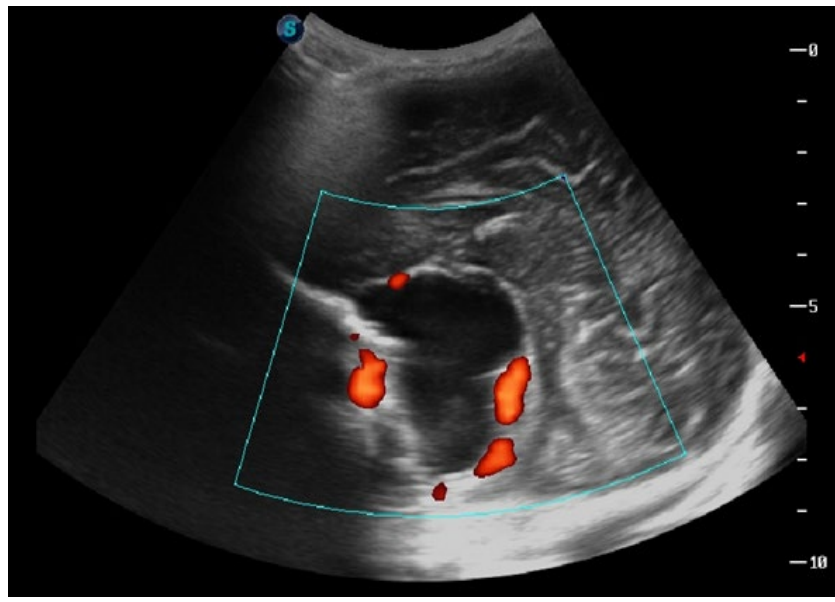

Fig. 4. Imagen sagital en línea media, con lesión quística de localización supraselar y prepontina, con desplazamiento posterior del tallo cerebral, y superior de tálamos y lóbulos frontales. Benavides M, Garcia M, 2020.

En la tabla 5 se muestra que el ultrasonido patológico fue más frecuente en el antecedente de Preeclampsia (3,3\%, 3 casos) e infección genitourinaria (2,2\%, 2 casos). No existió asociación estadísticamente significativa entre la complicación gestacional y el ultrasonido patológico ( $x 2$ de Yates= 0,00; 1 grado de libertad; $P=1,00)$. No se evidenció asociación estadísticamente significativa entre la edad gestacional y el ultrasonido patológico ( $x 2$ de Pearson= 0,69; 2 grados de libertad; $P=0,71)$.
Los diagnósticos clínicos que más se asociaron con un ultrasonido patológico fueron sepsis con punto de partida SNC con $6,7 \%$ (6 pacientes), seguido por la sepsis punto de partida a precisar en $5,6 \%$ de los casos (5 pacientes), e infección del SNC 2,2\% (2 pacientes). No existió asociación estadísticamente significativa entre las variables $(P>0,05)$.

El índice de resistencia de la arteria pericallosa en la totalidad de los pacientes tuvo un valor mínimo de 0,56, máximo de 0,85 , la mediana fue 0,68 , el percentil 25 se ubicó en 0,62 y el percentil 75 en 0,73 . En uno de los casos no fue posible la evaluación de la arteria pericallosa debido a la presencia de hidranencefalia.

No existieron diferencias estadísticamente significativas entre las medianas de la edad gestacional $(U=688,00 ; P=0,25)$ y del índice de resistencia de la arteria pericallosa $(U=628,00 ; P=$ $0,189)$, según el resultado del ultrasonido.

Se evidenciaron correlaciones negativas débiles y sin significancia estadística entre el índice de resistencia de la arteria pericallosa, la edad gestacional y la edad del producto de la concepción, como puede apreciarse en las figuras 5 y 6 .

Existió asociación estadísticamente significativa entre la prematuridad y la existencia de variante anatómica en el ecosonograma transfontanelar ( $X 2$ de Pearson $=6,26 ; 2$ grados de libertad; $P=0,04$ ).

Tabla 5. Asociación entre la complicación gestacional y hallazgos del ultrasonido.

\begin{tabular}{|c|c|c|c|c|c|c|}
\hline \multirow{3}{*}{ Complicación gestacional } & \multicolumn{4}{|c|}{ Hallazgo ultrasonido } & \multirow{2}{*}{\multicolumn{2}{|c|}{ Total }} \\
\hline & \multicolumn{2}{|c|}{ Normal } & \multicolumn{2}{|c|}{ Patológico } & & \\
\hline & f & $\%$ & f & $\%$ & f & $\%$ \\
\hline Cardiopatía & 1 & 1,1 & 0 & 0,0 & 1 & 1,1 \\
\hline Hipertensión arterial & 0 & 0,0 & 1 & 1,1 & 1 & 1,1 \\
\hline Eclampsia & 1 & 1,1 & 0 & 0,0 & 1 & 1,1 \\
\hline Preeclampsia & 4 & 4,4 & 3 & 3,3 & 7 & 7,7 \\
\hline Embarazo gemelar & 3 & 3,3 & 0 & 0,0 & 3 & 3,3 \\
\hline Infección genitourinaria & 11 & 12,2 & 2 & 2,2 & 13 & 14,4 \\
\hline Infección transmisión sexual (VDRL) & 3 & 3,3 & 0 & 0,0 & 3 & 3,3 \\
\hline Oligohidramnios & 2 & 2,2 & 0 & 0,0 & 2 & 2,2 \\
\hline Paludismo & 1 & 1,1 & 0 & 0,0 & 1 & 1,1 \\
\hline Epilepsia & 1 & 1,1 & 0 & 0,0 & 1 & 1,1 \\
\hline Síndrome convulsivo & 1 & 1,1 & 0 & 0,0 & 1 & 1,1 \\
\hline Trauma obstétrico & 0 & 0,0 & 1 & 1,1 & 1 & 1,1 \\
\hline Doble circular de cordón & 1 & 1,1 & 0 & 0,0 & 1 & 1,1 \\
\hline Edad materna avanzada (mayor 35 años) & 1 & 1,1 & 0 & 0,0 & 1 & 1,1 \\
\hline Sin complicaciones conocidas & 35 & 38,9 & 18 & 20,0 & 53 & 58,9 \\
\hline Total & 65 & 72,2 & 25 & 27,8 & 90 & 100 \\
\hline
\end{tabular}




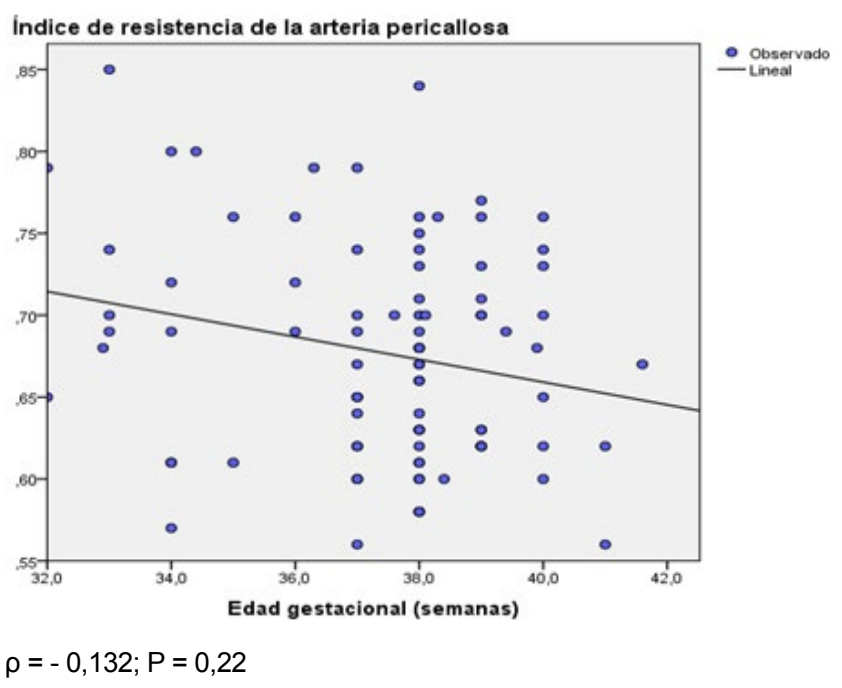

Fig. 5. Correlación entre el índice de resistencia de la arteria pericallosa y la edad gestacional.

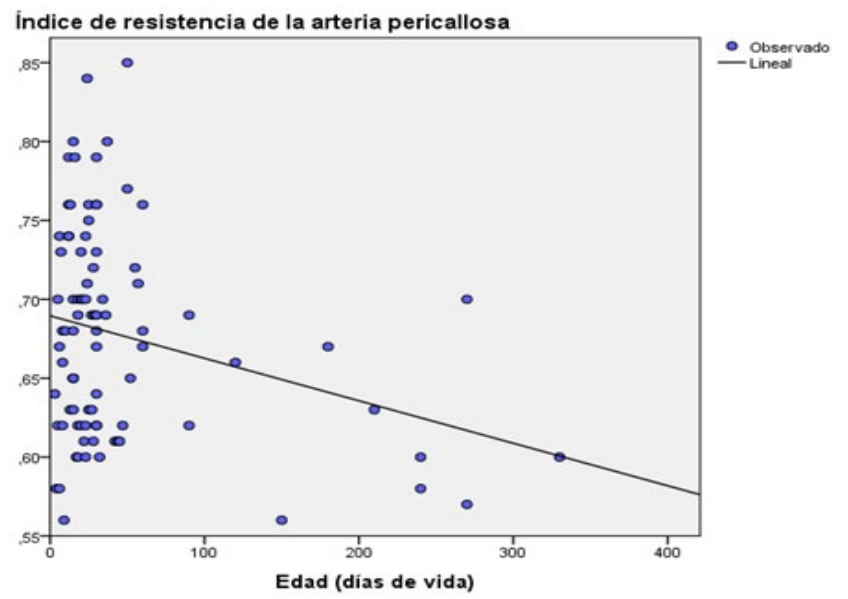

$\rho=-0,092 ; P=0,40$

Fig. 6. Correlación entre la edad del niño y el índice de resistencia de la arteria pericallosa.

\section{DISCUSIÓN}

De acuerdo con los resultados obtenidos predominó el sexo masculino con $58,9 \%$. Siendo el rango de edad destacado los neonatos y lactantes menores, entre 3 y 57 días de vida, $84,4 \%$. En su mayoría fueron pacientes a término con una mediana de 37 semanas, mientras que $23,3 \%$ de la muestra eran pacientes pretérminos. $42,2 \%$ presentaron antecedentes de complicaciones durante la etapa gestacional.

No sé evidenció asociación estadísticamente significativa entre ésta y el ultrasonido patológico. Hay que resaltar que de los antecedentes gestacionales patológicos de preeclampsia 7,7\% (7 pacientes), 3,3\% (3 pacientes) resultaron con UT patológico, hallazgo que coincide con el estudio de Cervantes et al., en 2012, en el que evaluaron a 64 pacientes con hemorragia de la matriz germinal, encontrando que $45 \%$ de los casos estaba asociada al antecedente materno de preeclampsia (14).
Con respecto a las variables de edad gestacional y hallazgos del ultrasonido, en contraste con otros estudios, la mayor cantidad de casos con hallazgos patológicos se encontraron en pacientes a término, tomando en cuenta que estos además representaban el mayor volumen de la muestra.

No existió asociación estadísticamente significativa entre el ultrasonido patológico y el diagnóstico clínico, ya que de toda la muestra con alto riesgo, $72 \%$ (65 casos) obtuvo un resultado normal, siendo patológicos $27,7 \%$ (25 casos), de estos los hallazgos más frecuentes fueron infección del SNC, sepsis con punto de partida en el SNC y sepsis punto de partida a precisar, de estos tres diagnósticos 14,4\% (13 pacientes) resultaron igualmente patológicos al ultrasonido transfontanelar.

Dentro de los hallazgos patológicos encontrados está la hemorragia de la matriz germinal $5,5 \%$ (5 casos), con degeneración quística subependimaria 1,1\% (1 caso), quistes subependimarios uni y bilaterales $7,7 \%$ (7 casos), hidrocefalia $4,4 \%$ (4 casos). Así como también se encontraron casos de hemorragia intraparenquimatosa. De las malformaciones congénitas fueron observadas agenesia del cuerpo calloso con colpocefalia, hidranencefalia, quiste aracnoideo. Asimismo, se evidenciaron ventriculitis, calcificaciones periventriculares, calcificaciones talámicas y vasculopatía lenticuloestriada.

En el estudio de Nagaraj et al., del 2016, evaluaron a 100 recién nacidos de alto riesgo, 38\% de ellos presentaron hallazgos anormales, $12 \%$ con hemorragia intracraneal, $13 \%$ hiperecogenicidad periventricular, $7 \%$ ventriculomegalia, $2 \%$ edema cerebral y $1 \%$ leucomalacia. Tres neonatos tuvieron hallazgos sugestivos de quiste simple en la fosa craneal media, agenesia del cuerpo calloso y quiste del plexo coroideo (16).

Otro estudio más reciente (2018), Diwakar y Khurana, analizaron 100 recién nacidos prematuros, en los que detectaron diferentes anomalías en la ecografía craneal que incluyen hidrocefalia $12 \%$, hemorragia intracraneal $6 \%$, edema cerebral $6 \%$, leucomalacia periventricular $2 \%$, quiste del plexo coroideo $1 \%$, septos intraventriculares $1 \%$ y colpocefalia $1 \%$ (17).

Shankar y Nithya, 2014, evaluaron la incidencia de anomalías en el ultrasonido cerebral (24\%) en neonatos de alto riesgo. $11 \%$ tenían evidencia de hemorragia intracraneal, $7 \%$ tálamos hiperecogénicos, 2\% EHI definida y 4\% tenían edema cerebral. Dos recién nacidos en el seguimiento regular habían desarrollado encefalomalacia quística con hidrocefalia (18).

En el presente estudio se encontró 3 casos de alteraciones en tálamos que van desde vasculopatía lenticuloestriada, calcificaciones y hemorragia intraparenquimatosa, los dos últimos en pacientes a término. En el 2007, Díaz et al., realizaron un estudio de 8 casos de lesiones talámicas. En él mencionan que la lesión talámica de tipo hemorrágica se presenta con mayor frecuencia en neonatos a término, coincidiendo con nuestro estudio. Observaron 2 casos de lesión talámica bilateral, ambos en neonatos a término y con antecedente de lesión hipóxico-isquémica severa. Lesión talámica unilateral en prematuros que no presentaron encefalopatía hipóxica isquémica, y tuvieron trastornos de la coagulación/plaquetopenia. No observaron factores predisponentes identificables en 3 neonatos (19). 
Los pacientes deben ser considerados como un todo, especialmente en la etapa neonatal, ya que pueden presentar tantos antecedentes de patología perinatal y la patología clínica, aumentando así mismo el factor de riesgo para presentar patologías cerebrales. Otro de los hallazgos frecuentes es el cavum de septum pellucidum, existiendo asociación estadísticamente significativa entre la prematuridad y la existencia de esta variante anatómica.

El índice de resistencia de la arteria pericallosa sin compresión obtuvo un valor mínimo de 0,56 , máximo de 0,85 , la mediana fue 0,68, el percentil 25 se ubicó en 0,62 y el percentil 75 en 0,73. A pesar de que se evidenció correlaciones negativas débiles y sin significancia estadística entre el índice de resistencia (IR) de la arteria pericallosa, la edad gestacional y la edad del producto de la concepción, en ambos casos se pudo constatar que a medida que aumenta tanto la edad gestacional, como la edad de vida extrauterina, se observa una leve disminución del índice de resistencia.

En el estudio de Zamora et al. del 2014, basado en pacientes menores de 3 meses, pretérmino y a término, el estudio de Pashaj del 2015, donde evaluaron a fetos entre las 18 y 41 semanas de gestación, el trabajo de Horgan et al., de 1989, que estudiaron 38 recién nacidos prematuros y a término asintomáticos, así como el estudio de Flink et al. del 2019, demostraron todos que los valores del IR disminuyen progresivamente con la edad gestacional, siendo más altos en los recién nacidos prematuros en comparación con los recién nacidos a término (20-23).

Al establecer los rangos de valores normales en todos los estudios varían ampliamente, por lo que puede ser necesario tomar en cuenta además de la edad gestacional y la edad de vida extrauterina, otros factores como el peso al nacer, entre otros.

Se puede concluir que el UT es el método de neuroimagen de elección para los recién nacidos de alto riesgo, siendo uno de los factores más importantes la posibilidad de evaluar el sistema nervioso central sin el uso de radiación ionizante, ni sedación del paciente. El ultrasonido permite detectar múltiples patologías, siendo uno de los objetivos principales la demostración o exclusión de hemorragia intracraneal en el recién nacido.

La edad gestacional y el diagnóstico clínico deben de ser tomados en cuenta a la hora de practicar el estudio, ya que pueden ser factores de riesgo en el desarrollo de lesiones cerebrales. Igualmente, el seguimiento mediante evaluaciones ultrasonográficas puede ser determinante para la caracterización evolutiva de lesiones diagnosticadas y de la mano con el médico clínico en el establecimiento de la correlación con la sintomatología neurológica, se logrará que en algunos casos no sea necesario el uso de otros métodos de imagen.

Por los hallazgos resultara necesario tomar en cuenta que todos los pacientes con antecedente gestacional materno de preeclapmsia deben ser evaluados de forma protocolizada mediante la realización de ultrasonido transfontanelar, debido a que estos pacientes tienen mayor posibilidad de mostrar alteraciones en el sistema nervioso central.
Es también pertinente mencionar la necesidad de contar con equipos de ultrasonido en las salas de UCIN para la evaluación de aquellos pacientes que por sus condiciones médicas no pueden ser trasladados a los servicios de imagen. Será necesario en próximos estudios la valoración específica de los pacientes con antecedente materno de preeclampsia, eclampsia y de aquellos con diagnóstico de desnutrición; así como la evaluación de los rangos de normalidad del índice de resistencia de la arteria pericallosa.

AGRADECIMIENTOS. Al Dr. Augusto Castroni y Dra. Laura Granella especialistas en radiología y neurología pediátrica respectivamente.

\section{REFERENCIAS BIBLIOGRÁFICAS}

1. Ares $S$, Díaz C. Seguimiento del recién nacido prematuro y del niño de alto riesgo biológico. Servicio de Neonatología. Hospital Universitario La Paz. Madrid. Pediatr Integral 2014; XVIII (6):344-355. Disponible en: https://www.pediatriaintegral. es/wp-content/uploads/2014/xviii06/01/344-355.pdf

2. Hechavarría L, Cruz U, Hernández M, López M. Protocolo de atención temprana a los neonatos con neuro-desarrollo de alto riesgo. Correo Científico Médico de Holguín. Cuba. 2018; (4):137-154. Disponible en: http://scielo.sld.cu/pdf/ccm/v22n1/ ccm12118.pdf

3. Ecury-Goossen G, Camfferman F, Leijser L, Govaert P, Dudink J. State of the Art Cranial Ultrasound Imaging in Neonates. J. Vis. Exp. 2015; e52238 (96):1-10. Disponible en: http://www. jove.com/video/52238

4. Riccabona M. Neonatal neurosonography. Eur J Radiol. 2014; 83 (9):1495-506. Disponible en: https://doi.org/10.1016/j. ejrad.2014.04.033

5. Venkatraman Bhat, Varun Bhat. Neonatal neurosonography: A pictorial essay. Indian J Radiol Imaging. 2014; 24(4):389-400.

6. Marchie T, Ayara C. Investigation of Infant Brain with or without Hydrocephalous in Our Environment Using Anterior Transfontanelle Ultrasound Scan. Niger J Surg. 2013; 19(1):712.

7. Choi S, Kim J, Ko J, Lee Y, Chang Y. Patterns of ischemic injury on brain images in neonatal group B Streptococcal meningitis. Korean J Pediatr. 2018; 61(8):245-252.

8. Llorens $\mathrm{R}$, Moreno A. EI ABC de la ecografía transfontanelar y más. Radiología. 2016; 58(S2):129-141.

9. Ciambra G, Arachi S, Protano C, Cellitti R, Caoci S, Di Biasi C $y$ cols. Accuracy of Transcranial Ultrasound in the Detection of Mild White Matter Lesions in Newborns. Neuroradiol j. 2013; 26(3):284-289.

10. Siegel M. Pediatric Sonography. 4th ed. Philadelphia: Lippincott Williams \& Wilkins; 2011

11. Rumack C, Wilson S, Charboneau J. Diagnostic Ultrasound. Vol 2. 4th Ed. Philadelphia: Mosby; 2016.

12. Timor-Tritsch I, Monteagudo A, Pilu G, Malinger D. Neuroecografía prenatal y neonatal. 2da ed. Madrid: Marbán; 2004.

13. Bruyn R. Pediatric Ultrasound: How, Why and When. 2nd ed. Londres: Churchill Livingstone; 2010. 
14. Cervantes M, Rivera M, Yescas G, Villegas R, Hernández G. Hemorragia intraventricular en recién nacidos pretérmino en una Unidad de Tercer Nivel en la Ciudad de México. Perinatol Reprod Hum 2012; 26 (1):17-24.

15. PorettiA, Huisman T. Neonatal Head and Spine Ultrasonography. Springer, 2015.

16. Nagaraj N, Berwal $P$, Srinivas A, Sehra R, Swami S, Jeevaji $P$ y cols. A study of neurosonogram abnormalities, clinical correlation with neurosonogram findings, and immediate outcome of high-risk neonates in Neonatal Intensive Care Unit. J Pediatr Neurosci. 2016; 11(3):200-205.

17. Diwakar R, Khurana O. Cranial sonography in preterm infants with short review of literature. J Pediatr Neurosci 2018; 13(2):141-149.

18. Shankar $P$, Nithya $S$. Role of cranial ultrasound in high-risk neonates in NICU. J Evol Med Dent Sci 2014; 3(15):3970-3976.

19. Díaz $H$, Hernández $C$, Huamán J. Lesiones talámicas en neonatos evaluadas por ultrasonido transfontanelar: casos reportados en cinco años en el Instituto Materno Perinatal, Lima, Perú. Acta Médica Peruana, 2007; 24(2):101-104.
20. Zamora C, Tekes A, Alqahtani E, Kalayci O, Northington $F$, Huisman T. Variability of resistive indices in the anterior cerebral artery during fontanel compression in preterm and term neonates measured by transcranial duplex sonography. J Perinatol 2014; 34:306-310.

21. Pashaj S, Merz E, Wellek S. Normal doppler reference values of the pericallosal artery. Ultraschall in Med 2015; 36(4):375-380.

22. Horgan J, Rumack C, Hay T, Manco-Johnson M, Merenstein G, Esola C. Absolute intracranial blood-flow velocities evaluated by duplex doppler sonography in asymptomatic preterm and term neonates. Am J Roentgenol 1989; 152:1059-1064.

23. Flink A, Sandgren T, Ford K, Rosenberg J, Ringertz H, Barth R $y$ col. Normal values of the resistivity index of the pericallosal artery with and without compression of the anterior fontanelle. Pediatr Radiol. 2019; 49(5):646-651. 